\title{
Temporal variation of the phytoplankton community at short sampling intervals in the Mundaú reservoir, Northeastern Brazil
}

\author{
Ênio Wocyli Dantas ${ }^{1,2,3,6}$, Ariadne do Nascimento Moura², Maria do Carmo Bittencourt-Oliveira ${ }^{3}$, \\ João Dias de Toledo Arruda Neto ${ }^{4,5}$ and Airlton de Deus C. Cavalcanti ${ }^{2}$
}

Received: July 19, 2007. Accepted: January 31, 2008

\begin{abstract}
RESUMO - (Variação temporal da comunidade fitoplanctônica em curtos intervalos amostrais no reservatório de Mundaú, Nordeste brasileiro). O objetivo deste estudo foi detectar a influência dos fatores abióticos sobre a comunidade fitoplanctônica em um reservatório de abastecimento, em curtos intervalos amostrais. As amostras foram coletadas na subsuperfície $(0,1 \mathrm{~m})$ e fundo das regiões pelágica ( $8 \mathrm{~m}$ ) e litorânea $(2 \mathrm{~m})$, durante dois períodos sazonais, estações seca e chuvosa. As variáveis abióticas analisadas foram: temperatura da água, oxigênio dissolvido, condutividade elétrica, sólidos totais dissolvidos, turbidez, pH, nitrogênio total, nitrito, nitrato, fósforo total, fósforo total dissolvido e ortofosfato. A biomassa fitoplanctônica foi determinada a partir de valores de biovolume. A influência das variáveis abióticas na dinâmica das espécies fitoplanctônicas foi determinada através da Análise de Correspondência Canônica. A biomassa algal variou de $1,17 \times 10^{4}$ a $9,21 \times 10^{4} \mu \mathrm{g} . \mathrm{L}^{-1}$, sendo que as cianobactérias apresentaram valores de biomassa entre $1,07 \times 10^{4} \mathrm{e}$ $8,21 \times 10^{4} \mu \mathrm{g} \cdot \mathrm{L}^{-1}$. A mais alta disponibilidade de fósforo, limitação de nitrogênio, $\mathrm{pH}$ alcalino e estabilidade térmica favoreceram florações de cianobactérias, especialmente durante o período seco. As diferenças entre os horários e as estações amostrais na distribuição da comunidade fitoplanctônica foram determinadas pelos valores de temperatura, $\mathrm{pH}$, fósforo total e turbidez. As associações fitoplanctônicas que mais se destacaram foram do grupo funcional $\mathrm{Sn}$, formado por Cylindrospermopsis raciborskii, que predominou no sistema em ambos períodos sazonais. Concluiu-se que o fitoplâncton apresentou uma forte influência das variáveis abióticas especialmente no estabelecimento de padrões sazonais de distribuição.
\end{abstract}

Palavras-chave: Cylindrospermopsis raciborskii, eutrofização, nutrientes, reservatório de abastecimento

ABSTRACT - (Temporal variation of the phytoplankton community at short sampling intervals in the Mundaú reservoir, Northeastern Brazil). The aim of this study was to determine how abiotic factors drive the phytoplankton community in a water supply reservoir within short sampling intervals. Samples were collected at the subsurface $(0.1 \mathrm{~m})$ and bottom of limnetic $(8 \mathrm{~m})$ and littoral $(2 \mathrm{~m})$ zones in both the dry and rainy seasons. The following abiotic variables were analyzed: water temperature, dissolved oxygen, electrical conductivity, total dissolved solids, turbidity, $\mathrm{pH}$, total nitrogen, nitrite, nitrate, total phosphorus, total dissolved phosphorus and orthophosphate. Phytoplankton biomass was determined from biovolume values. The role abiotic variables play in the dynamics of phytoplankton species was determined by means of Canonical Correspondence Analysis. Algae biomass ranged from $1.17 \times 10^{4}$ to $9.21 \times 10^{4} \mu \mathrm{g}$. $\mathrm{L}^{-1}$; cyanobacteria had biomass values ranging from $1.07 \times 10^{4}$ to $8.21 \times 10^{4} \mu \mathrm{g}$. $\mathrm{L}^{-1}$. High availability of phosphorous, nitrogen limitation, alkaline $\mathrm{pH}$ and thermal stability all favored cyanobacteria blooms, particularly during the dry season. Temperature, $\mathrm{pH}$, total phosphorous and turbidity were key factors in characterizing the phytoplankton community between sampling times and stations. Of the species studied, Cylindrospermopsis raciborskii populations were dominant in the phytoplankton in both the dry and rainy seasons. We conclude that the phytoplankton was strongly influenced by abiotic variables, particularly in relation to seasonal distribution patterns.

Key words: Cylindrospermopsis raciborskii, eutrophication, nutrients, water supply reservoir

\section{Introduction}

Reservoirs are continental environments generally viewed as a transition between lotic and lentic systems, as they are manmade lakes used to fulfill specific needs
(Straskraba \& Tundisi 2000). These artificial ecosystems exhibit space/time variability affecting the formation of several ecological niches. The ability or potential algae have to comply with habitat heterogeneity is a result of their behavior and reproduction characteristics. Thus,

\footnotetext{
1 Universidade Estadual da Paraíba, Centro de Ciências Biológicas e Sociais Aplicadas, Campus V, Av. das Baraúnas 352, João Pessoa, PB, Brasil

2 Universidade Federal Rural de Pernambuco, Departamento de Biologia, Área de Botânica, Rua D. Manoel de Medeiros s.n., 52171-030 Recife, PE, Brasil (ariadne@db.ufrpe.br)

3 Escola Superior de Agricultura "Luiz de Queiroz", Departamento de Ciências Biológicas, Av. Pádua Dias 11, C. Postal 9, 13418-900 Piracicaba, SP, Brasil

4 Universidade de São Paulo, Instituto de Física, Rua do Matão, Travessa R 187, 05508-090 Cidade Universitária, SP, Brasil

5 Universidade de Santo Amaro, Rua Prof. Enéas de Siqueira Neto 340, Jardim das Imbuias, 04829-300 São Paulo, SP, Brasil

6 Corresponding Author: eniowocyli@yahoo.com.br
} 
phytoplankton is important in predicting environmental events and is a much-needed input in interpreting the functioning of reservoirs.

The small shift in daytime length in tropical environments does not impede seasonal changes in phytoplankton communities. Fluctuations in biomass and phytoplankton composition are mainly related to hydrological changes (Huszar \& Reynolds 1997). Shortterm studies are important to the understanding of processes occurring on short time scales, such as atelomixis, deoxygenating and denitrification, which contribute toward changes in the dynamics and structure of phytoplankton communities.

In Brazil, studies on phytoplankton in short sampling intervals ( 24 hours) have been carried out in systems of the southeastern region (Ramirez \& Bicudo 2002; 2005; Lopes et al. 2005) and northern region (Huszar \& Reynolds 1997; Melo \& Huszar 2000), which exhibit distinct climate characteristics from the northeastern region. In Brazilian lakes and reservoirs studied in 24 hour sampling intervals, temperature plays an important role in the structure and dynamics of the phytoplankton community, explaining the space and time variability of algae throughout a daily cycle.

The northeastern region of Brazil is mainly characterized by its semi-arid climate, where water shortages reflect the temporary aspect of its hydrographic basins and the low precipitation, typically below $1.000 \mathrm{~mm} \cdot \mathrm{y}^{-1}$ (Strahler 1986). The construction of reservoirs is a solution to problems associated to water needs, assuring availability in the dry season. However, influencing factors such as long water residence time in the reservoirs, high temperatures and evapotranspiration, along with an ever increasing dumping of domestic and industrial sewage from urban centers, have inflicted environmental problems such as eutrophication on reservoirs located in northeastern Brazil. In the region and particularly in the state of Pernambuco, $90 \%$ of all reservoirs are eutrophic (Bouvy et al. 2000) and algae blooms occur frequently in a number of aquatic systems. The most common cyanobacteria blooms are from Cylindrospermopsis raciborskii (Woloszynska) Seenayya \& Subba Raju (Bouvy et al. 1999; 2000; 2001), Microcystis aeruginosa (Kützing) Kützing (Chellappa \& Costa 2003) and Anabaena spiroides Klebahn (Molica et al. 2005).

There are studies demonstrating that, under eutrophic conditions, phytoplankton biomass is controlled by abiotic factors (the bottom-up effect); small variations in nutrient levels within short sampling intervals result in a rapid change in the structure of the algae community. However, there is a lack of studies using short sampling intervals and associating environmental conditions of aquatic systems in northeastern Brazil with the phytoplankton community. Thus, the aim of the present study was to detect the influence of abiotic variables on temporal distributions (daily and between the dry and rainy seasons) of a phytoplankton community in a eutrophic drinking water supply system (the Mundaú Reservoir) in northeastern Brazil during short periods.

\section{Material and methods}

The Mundaú reservoir lies within the coordinates $08^{\circ} 56^{\prime} 47^{\prime \prime} \mathrm{S}$ and $36^{\circ} 29^{\prime} 33^{\prime \prime} \mathrm{W}$ at an altitude of $716 \mathrm{~m}$. Maximum reservoir depths are 10 and 9 meters in the rainy and dry seasons, respectively. The accumulation capacity of this reservoir is $1.968 .000 \mathrm{~m}^{3}$ and the water retention time coincides with the duration of the dry season.

The occurrence of macrophytes is not important, but isolated banks of Nymphaea spp have been observed. The terrestrial vegetation around the reservoir is made up of species featured in the caatinga (xeric scrubland and thorn forest). This reservoir is intended for the public water supply, but currently receives part of the urban drainage from the city of Garanhuns, Pernambuco (SHR 2000).

Samples were collected in two seasonal periods: dry (January 2005) and rainy (June 2005) over 20 hour sampling periods during daylight ( $8 \mathrm{am}, 12 \mathrm{pm}$ and $4 \mathrm{pm})$ and dark ( 8 pm, 12 am and 4 am) hours. Samples were collected from the subsurface in pelagic $\left(\mathrm{E}_{1}\right)$ and littoral $\left(\mathrm{E}_{2}\right)$ zones using a van Dorn bottle. Samplings were performed at $8 \mathrm{~m}$ and $2 \mathrm{~m}$, respectively.

Abiotic variables were determined in situ and included water temperature and dissolved oxygen using an oximeter (Schott Glaswerke Mainz, handylab OX1); electrical conductivity and total dissolved solids (TSD) by means of a conductivimeter (Schott Glaswerke Mainz, handylab LF1); turbidity using a turbidimeter (Hanna Instruments, HI 93703), and pH using a potentiometer (Digimed, DMPH-2). Water transparency was determined using a $25 \mathrm{~cm}$ diameter Secchi disc. The determination of the photic zone was performed following the procedure described in Margalef (1983).

Samples were collected at two depths in each of the sampling seasons using a Van Dorn bottle for subsequent laboratory analyses of dissolved and total nutrients. The water was conditioned in $300 \mathrm{~mL}$ polyethylene flasks and kept refrigerated until the analysis. Samples were filtered through $47 \mathrm{~mm}$ AP20 glass multi-pore filters for the determination of dissolved nutrients. Non-filtered aliquots were used for the determination of total nutrients. Analysis to determine concentrations of total nitrogen ( $\mu$ g.TN.L $\left.{ }^{-1}\right)$, nitrite 
$\left(\mu \mathrm{g} \cdot \mathrm{N}-\mathrm{NO}_{2} \cdot \mathrm{L}^{-1}\right)$ and nitrate $\left(\mu \mathrm{g} . \mathrm{N}-\mathrm{NO}_{3} \cdot \mathrm{L}^{-1}\right)$ followed procedures described in Valderrama (1981), Mackereth et al. (1978) and Golterman et al. (1971), respectively. Total phosphorus ( $\mu$ g.TP.L ${ }^{-1}$ ) and total dissolved phosphorus $\left(\mu \mathrm{g}\right.$.TDP.L $\left.{ }^{-1}\right)$ were determined following Valderrama (1981). Orthophosphate ( $\left.\mu \mathrm{g} . \mathrm{P}-\mathrm{PO}_{4} \cdot \mathrm{L}^{-1}\right)$ was determined following Strickland \& Parsons (1965). The content of particulate phosphorus $\left(\mu \mathrm{g}\right.$. PP.L $\left.^{-1}\right)$ and dissolved organic phosphorus ( $\mu$ g.DOP. $L^{-1}$ ) were determined from the difference between TP and TDP, and PTD and $\mathrm{P}-\mathrm{PO}_{4}$, respectively.

Samples for taxonomic analysis were preserved in $4 \%$ formaldehyde. Identification was performed down to species level using an optical microscope (Zeiss/ Axioskop) or to the highest possible taxonomic resolution using relevant literature (Komárek \& Foot 1983; Sant'Anna 1984; Round et al. 1990; Komárek \& Anagnostidis 1999; Komárek \& Anagnostidis 2005).

Water aliquots of $100 \mathrm{~mL}$ were collected daily from the reservoir and preserved in acetic Lugol's solution for the determination of cell density (cell. $\mathrm{L}^{-1}$ ) following the Utermöhl method (Hasle 1978). Densities were converted into biovolumes following the procedure in Edler (1979).

Analysis of variance (ANOVA) was calculated using the BioEstat 3.0 statistical package (Ayres et al. 2003) and employed to determine the degree of temporal variation (time of the day and seasonal period) and spatial variation (depth and sampling station). The significance level was set at 5\%. Canonical Correspondence Analysis using the PC-ORD software version 4.14 for Windows (McCune \& Mefford 1999) was employed for the evaluation of the temporal gradient and to determine what abiotic variables were influencing the dynamics of phytoplankton species.

\section{Results}

In the dry period, the Mundaú reservoir exhibited lower oxygenated water, higher $\mathrm{pH}$ in the subsurface and lower turbidity when compared to the rainy season, which had well-oxygenated water, $\mathrm{pH}$ ranging from near neutral to lower values and high turbidity, especially near the sediment in the pelagic zone (Tab. 1, 2).

No vertical differences were observed $(p>0.05)$ in the dry season regarding temperature, dissolved oxygen, electrical conductivity, total dissolved solids, turbidity and $\mathrm{pH}$. A difference in turbidity $(\mathrm{F}=15.850$, $p<0.01$ ) was observed between the light and dark hours; higher turbidity was found in the dark hours, except in $\mathrm{E}_{2}$ at a depth of $2 \mathrm{~m}$ (Tab. 1). Electrical conductivity and total dissolved solids had lower variation coefficients $(0.38 \%$ and $0.31 \%)$, with no vertical or temporal variability during light and dark hours.

The most significant vertical differences in the rainy season were observed in $\mathrm{E}_{1}$, where temperature $(\mathrm{F}=15.180, p<0.01)$, dissolved oxygen $(\mathrm{F}=89.497$, $p<0.001)$, electrical conductivity $(\mathrm{F}=31.235$, $p<0.001)$, total dissolved solids ( $\mathrm{F}=36.577, p<0.001)$ and $\mathrm{pH}(\mathrm{F}=32.216, p<0.001)$ had higher values at the subsurface. No differences were observed between light and dark hours in the rainy season, with the exception of the concentrations of dissolved oxygen at the subsurface of the pelagic zone $(\mathrm{F}=28.880, p<0.01)$, which were higher during dark hours.

The analysis of nutrients from the dry season (Tab. 1) as a function of depth revealed differences in the concentrations of total nitrogen $(\mathrm{F}=10.026, p<0.01)$ and total dissolved phosphorus $(\mathrm{F}=19.267, p<0.001)$ at both stations as well as orthophosphate and total phosphorus, with higher values at the bottom $(\mathrm{p}<0.01)$, particularly in $\mathrm{E}_{1}$. Vertical differences for $\mathrm{NO}_{3}$ and $\mathrm{NO}_{2}$ were registered only during daylight hours, with the smaller values occurring at the subsurface, except for the $\mathrm{NO}_{2}$ concentrations in $\mathrm{E}_{2}$. These two nitrogen rich forms also displayed horizontal variation between light and dark hours

$\mathrm{NO}_{3}$ levels were higher in $\mathrm{E}_{1}$, whereas $\mathrm{NO}_{2}$ levels were higher in $\mathrm{E}_{2}$. $\mathrm{NO}_{3}$ concentrations were higher during dark hours at the subsurface and during light hours at the bottom. $\mathrm{NO}_{2}$ levels between light/dark hours exhibited a similar pattern in $E_{1}$, but an opposite pattern in $E_{2}$.

There was an increase in nitrogen-composed products, total nitrogen, nitrate and nitrite (Tab. 2) in the rainy season as well as a reduction in dissolvedphosphate elements, total dissolved phosphorus, orthophosphate and dissolved organic phosphorus (Tab. 2). Vertical differences were significant only in $\mathrm{E}_{1}$, with total nitrogen levels, nitrate, nitrite and total phosphorus significantly higher $(\mathrm{p}<0.01)$ near the sediment. Horizontal differences between light/dark hours were more evident for total nitrogen and orthophosphate concentrations. These two nutrients had higher values at the subsurface in the dark hours. Total nitrogen was higher concentrations in $\mathrm{E}_{2}$, whereas orthophosphate was higher in $\mathrm{E}_{1}$.

Biotic variables - Phytoplankton flora was represented by 66 infrageneric taxa and six generic taxa. The highest species richness was observed for Chlorophyta, corresponding to $54.17 \%$ of the infrageneric taxa identified, followed by Cyanophyta (19.44\%), Bacillariophyta (15.28\%), Euglenophyta (8.33\%), Dinophyta (1.39\%) and Cryptophyta (1.39\%) (Tab. 3).

Phytoplankton biomass ranged from $1.17 \times 10^{4}$ to $9.21 \times 10^{4} \mu \mathrm{g} . \mathrm{L}^{-1}$ (Fig. 1). The community was made up of Bacillariophyta, Chlorophyta, Cryptophyta, 
Table 1. Physical and chemical variables of the Mundaú reservoir, Northeastern Brazil at the sampling stations $\mathrm{E}_{1}$ e $\mathrm{E}_{2}$ during the dry season. (-) dates without sampling.

\begin{tabular}{|c|c|c|c|c|c|c|c|c|c|c|c|c|c|c|c|c|}
\hline & \multicolumn{8}{|c|}{$\mathrm{E}_{1}$} & \multicolumn{8}{|c|}{$\mathrm{E}_{2}$} \\
\hline & \multicolumn{4}{|c|}{$0.1 \mathrm{~m}$} & \multicolumn{4}{|c|}{$8 \mathrm{~m}$} & \multicolumn{4}{|c|}{$0.1 \mathrm{~m}$} & \multicolumn{4}{|c|}{$2 \mathrm{~m}$} \\
\hline & \multicolumn{2}{|c|}{ Light } & \multicolumn{2}{|c|}{ Dark } & \multicolumn{2}{|c|}{ Light } & \multicolumn{2}{|c|}{ Dark } & \multicolumn{2}{|c|}{ Light } & \multicolumn{2}{|c|}{ Dark } & \multicolumn{2}{|c|}{ Light } & \multicolumn{2}{|c|}{ Dark } \\
\hline & Mean & $\mathrm{SD}$ & Mean & $\mathrm{SD}$ & Mean & $\mathrm{SD}$ & Mean & $\mathrm{SD}$ & Mean & $\mathrm{SD}$ & Mean & $\mathrm{SD}$ & Mean & $\mathrm{SD}$ & Mean & $\mathrm{SD}$ \\
\hline $\operatorname{Secchi}(\mathrm{m})$ & 0.3 & 0.0 & - & - & - & - & - & - & 0.4 & 0.0 & - & - & - & - & - & - \\
\hline $\mathrm{T}^{\circ} \mathrm{C}$ & 27.5 & 0.8 & 27.3 & 0.4 & 25.4 & 0.5 & 25.3 & 0.2 & 28.3 & 0.6 & 27.5 & 0.8 & 27.2 & 1.3 & 26.9 & 0.4 \\
\hline Dissolved oxygen $\left(\mathrm{mg} \cdot \mathrm{L}^{-1}\right)$ & 0.8 & 0.4 & 1.2 & 1.1 & 0.2 & 0.2 & 0.4 & 0.2 & 0.8 & 0.0 & 1.8 & 1.1 & 0.7 & 0.1 & 1.2 & 1.0 \\
\hline Electric conductivity $\left(\mathrm{uS} . \mathrm{cm}^{-1}\right)$ & 396.3 & 0.6 & 398.3 & 1.5 & 393.7 & 3.5 & 394.7 & 3.5 & 396.0 & 1.7 & 398.0 & 1.0 & 392.0 & 2.0 & 390.3 & 2.3 \\
\hline TDS $\left(m g . L^{-1}\right)$ & 349.3 & 0.6 & 350.7 & 1.2 & 346.3 & 2.1 & 347.0 & 3.0 & 349.0 & 1.7 & 351.3 & 0.6 & 345.7 & 1.5 & 343.7 & 2.1 \\
\hline Turbidity (NTU) & 32.9 & 1.4 & 35.9 & 2.2 & 21.2 & 5.0 & 26.3 & 5.1 & 32.9 & 1.4 & 36.6 & 1.2 & 32.5 & 4.6 & 29.9 & 3.9 \\
\hline $\mathrm{pH}$ & 8.3 & 0.1 & 8.3 & 0.2 & 7.2 & 0.1 & 7.2 & 0.1 & 8.4 & 0.1 & 8.2 & 0.1 & 7.9 & 0.4 & 7.6 & 0.3 \\
\hline $\mathrm{TN}\left(\mu \mathrm{g} \cdot \mathrm{L}^{-1}\right)$ & 57.4 & 11.4 & 61.9 & 5.4 & 102.7 & 22.3 & 81.6 & 13.4 & 61.9 & 22.7 & 59.9 & 8.9 & 70.5 & 30.2 & 80.1 & 20.4 \\
\hline $\mathrm{NO}_{3}\left(\mu \mathrm{g} \cdot \mathrm{L}^{-1}\right)$ & 165.7 & 35.9 & 214.1 & 43.1 & 331.5 & 149.4 & 214.1 & 72.8 & 131.2 & 97.9 & 172.6 & 83.7 & 179.5 & 83.7 & 179.5 & 97.9 \\
\hline $\mathrm{NO}_{2}\left(\mu \mathrm{g} \cdot \mathrm{L}^{-1}\right)$ & 8.2 & 0.5 & 13.8 & 3.6 & 42.6 & 48.7 & 8.2 & 2.4 & 39.2 & 39.6 & 20.4 & 16.0 & 8.5 & 0.9 & 23.2 & 26.4 \\
\hline $\mathrm{TP}\left(\mu \mathrm{g} . \mathrm{L}^{-1}\right)$ & 106.5 & 5.2 & 104.8 & 10.5 & 147.2 & 17.3 & 130.0 & 19.8 & 95.6 & 14.6 & 110.0 & 10.5 & 117.4 & 17.2 & 105.4 & 2.0 \\
\hline TDP $\left(\mu \mathrm{g} \cdot \mathrm{L}^{-1}\right)$ & 54.9 & 7.8 & 49.8 & 9.7 & 73.1 & 7.8 & 73.6 & 7.3 & 49.2 & 8.6 & 56.5 & 4.5 & 60.6 & 5.6 & 60.6 & 3.1 \\
\hline $\mathrm{PO}_{4}\left(\mu \mathrm{g} \cdot \mathrm{L}^{-1}\right)$ & 75.2 & 2.7 & 70.6 & 9.6 & 76.7 & 7.0 & 92.0 & 8.0 & 67.5 & 5.3 & 75.2 & 5.3 & 69.0 & 4.6 & 69.0 & 20.1 \\
\hline $\mathrm{PP}^{4}\left(\mu \mathrm{g} \cdot \mathrm{L}^{-1}\right)$ & 51.6 & 13.0 & 55.0 & 7.2 & 74.1 & 9.6 & 56.4 & 25.6 & 46.4 & 12.0 & 53.5 & 9.4 & 56.8 & 21.3 & 44.7 & 3.7 \\
\hline $\mathrm{DOP}\left(\mu \mathrm{g} \cdot \mathrm{L}^{-1}\right)$ & 54.9 & 7.8 & 26.7 & 8.6 & 48.1 & 6.5 & 43.6 & 7.0 & 27.2 & 9.4 & 32.0 & 5.6 & 38.1 & 7.1 & 38.1 & 7.6 \\
\hline $\mathrm{N}: \mathrm{P}$ & 1.2 & 0.3 & 1.3 & 0.2 & 1.5 & 0.3 & 1.4 & 0.1 & 1.4 & 0.3 & 1.2 & 0.3 & 1.4 & 0.8 & 1.7 & 0.5 \\
\hline
\end{tabular}

Table 2. Physical and chemical variables of the Mundaú Reservoir, Northeastern Brazil at the sampling stations $\mathrm{E}_{1}$ e $\mathrm{E}_{2}$ during the rainy season. (-) dates without sampling

\begin{tabular}{|c|c|c|c|c|c|c|c|c|c|c|c|c|c|c|c|c|}
\hline & \multicolumn{8}{|c|}{$\mathrm{E}_{1}$} & \multicolumn{8}{|c|}{$\mathrm{E}_{2}$} \\
\hline & \multicolumn{4}{|c|}{$0.1 \mathrm{~m}$} & \multicolumn{4}{|c|}{$8 \mathrm{~m}$} & \multicolumn{4}{|c|}{$0.1 \mathrm{~m}$} & \multicolumn{4}{|c|}{$2 \mathrm{~m}$} \\
\hline & \multicolumn{2}{|c|}{ Light } & \multicolumn{2}{|c|}{ Dark } & \multicolumn{2}{|c|}{ Light } & \multicolumn{2}{|c|}{ Dark } & \multicolumn{2}{|c|}{ Light } & \multicolumn{2}{|c|}{ Dark } & \multicolumn{2}{|c|}{ Light } & \multicolumn{2}{|c|}{ Dark } \\
\hline & Mean & $\mathrm{SD}$ & Mean & $\overline{\mathrm{SD}}$ & Mean & $\mathrm{SD}$ & Mean & $\mathrm{SD}$ & Mean & SD & Mean & $\mathrm{SD}$ & Mean & $\mathrm{SD}$ & Mean & $\mathrm{SD}$ \\
\hline Secchi $(\mathrm{m})$ & 0.3 & 0.0 & - & - & - & - & - & - & 0.3 & 0.0 & - & - & - & - & - & - \\
\hline $\mathrm{T}^{\circ} \mathrm{C}$ & 23.6 & 0.2 & 23.3 & 0.2 & 22.9 & 0.4 & 22.7 & 0.2 & 23.8 & 0.3 & 23.2 & 0.2 & 23.5 & 0.2 & 23.0 & 0.2 \\
\hline Dissolved oxygen (mg.L $\left.\mathrm{L}^{-1}\right)$ & 6.3 & 0.4 & 7.7 & 0.1 & 1.2 & 0.9 & 1.8 & 1.6 & 6.4 & 0.3 & 6.3 & 1.2 & 6.1 & 0.8 & 5.9 & 1.1 \\
\hline Electric conductivity $\left(\mathrm{uS} . \mathrm{cm}^{-1}\right)$ & 755.3 & 110.9 & 690.3 & 24.2 & 526.0 & 26.9 & 537.3 & 26.3 & 664.0 & 3.5 & 681.3 & 20.6 & 667.0 & 2.0 & 685.0 & 22.1 \\
\hline TDS (mg.L-1 $)$ & 678.7 & 90.7 & 626.0 & 22.3 & 476.7 & 24.5 & 485.7 & 23.3 & 602.3 & 3.8 & 617.7 & 18.6 & 605.3 & 1.2 & 621.7 & 20.0 \\
\hline Turbidity (NTU) & 53.5 & 8.2 & 55.2 & 7.3 & 433.7 & 163.3 & 362.3 & 70.5 & 39.1 & 10.5 & 41.2 & 11.6 & 50.0 & 7.5 & 34.5 & 15.2 \\
\hline $\mathrm{pH}$ & 7.3 & 0.2 & 7.3 & 0.1 & 6.8 & 0.1 & 6.9 & 0.2 & 7.1 & 0.0 & 7.1 & 0.2 & 7.2 & 0.4 & 7.2 & 0.3 \\
\hline $\mathrm{TN}\left(\mu \mathrm{g} . \mathrm{L}^{-1}\right)$ & 88.4 & 11.9 & 162.6 & 46.0 & 242.4 & 22.0 & 201.6 & 8.4 & 136.7 & 22.0 & 183.1 & 37.5 & 210.9 & 83.5 & 168.8 & 81.6 \\
\hline $\mathrm{NO}\left(\mu \mathrm{g} . \mathrm{L}^{-1}\right)$ & 234.4 & 44.2 & 259.8 & 20.6 & 769.7 & 57.8 & 621.2 & 15.5 & 283.3 & 63.2 & 279.4 & 20.6 & 277.4 & 23.7 & 304.8 & 25.5 \\
\hline $\mathrm{NO}_{2}\left(\mu \mathrm{g} . \mathrm{L}^{-1}\right)$ & 52.8 & 13.5 & 71.3 & 3.3 & 199.4 & 25.7 & 195.7 & 27.7 & 62.6 & 22.9 & 70.2 & 9.5 & 67.7 & 23.3 & 73.4 & 12.6 \\
\hline $\mathrm{TP}^{2}\left(\mu \mathrm{g} \cdot \mathrm{L}^{-1}\right)$ & 73.3 & 7.8 & 82.8 & 4.0 & 298.3 & 38.1 & 311.7 & 59.6 & 88.0 & 13.9 & 87.6 & 12.3 & 84.5 & 12.8 & 88.0 & 11.8 \\
\hline TDP $\left(\mu \mathrm{g} . \mathrm{L}^{-1}\right)$ & 28.2 & 14.5 & 29.8 & 19.6 & 21.0 & 2.5 & 24.2 & 15.1 & 17.1 & 2.7 & 27.4 & 4.8 & 17.9 & 3.1 & 20.6 & 7.0 \\
\hline $\mathrm{PO}_{4}\left(\mu \mathrm{g} \cdot \mathrm{L}^{-1}\right)$ & 24.9 & 3.6 & 61.5 & 37.0 & 39.1 & 10.7 & 29.6 & 31.0 & 14.3 & 3.4 & 41.4 & 30.6 & 26.0 & 12.5 & 35.5 & 12.3 \\
\hline $\mathrm{PP}\left(\mu \mathrm{g} . \mathrm{L}^{-1}\right)$ & 45.1 & 22.4 & 53.0 & 15.9 & 277.2 & 40.2 & 287.5 & 59.7 & 70.9 & 15.4 & 60.2 & 7.9 & 66.7 & 13.4 & 67.4 & 8.7 \\
\hline DOP $\left(\mu \mathrm{g} . \mathrm{L}^{-1}\right)$ & 20.1 & 13.4 & 9.7 & 8.6 & 8.3 & 5.9 & 14.6 & 6.2 & 12.4 & 1.9 & 13.9 & 5.3 & 9.4 & 7.1 & 9.1 & 6.7 \\
\hline $\mathrm{N}: \mathrm{P}$ & 2.7 & 0.4 & 4.4 & 1.4 & 1.8 & 0.4 & 1.5 & 0.2 & 3.5 & 0.6 & 4.7 & 1.2 & 5.5 & 1.7 & 4.3 & 2.1 \\
\hline
\end{tabular}



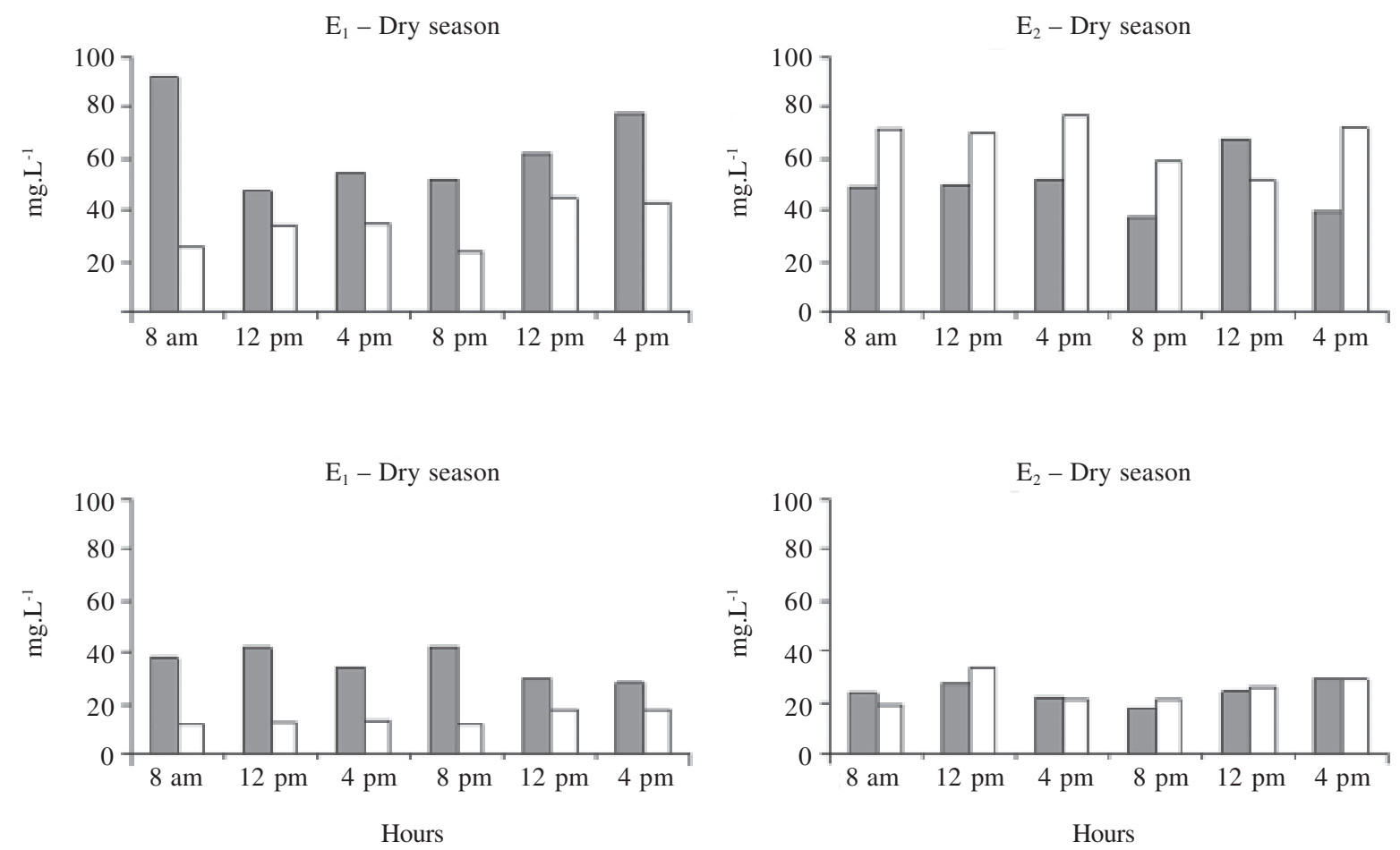

Figure 1. Spatial and temporal variations of phytoplanktonic biomass in the Mundaú reservoir, Northeastern Brazil.

Cyanophyta, Dinophyta and Euglenophyta. Cyanophyta - the dominant group (Tab. 3), with more than $80 \%$ of total biomass - was the only group displaying significant differences in depth, particularly in $\mathrm{E}_{1}$. Biomass values were significantly higher at the subsurface $(\mathrm{F}=18.255$, $p<0.01)$. There were differences in total biomass between seasons, with less biomass in the rainy season (Fig. 1). In the dry season, the increase of phytoplankton biomass occurred beginning at 12 am, reaching its highest values at the first daylight, especially in $E_{1}$ (Fig. 1). Higher biomass in the rainy season was registered in $\mathrm{E}_{1}$ during daylight hours until $8 \mathrm{pm}$, with a reduction beginning at 12 am to 4 am (Fig. 1).

Cylindrospermopsis raciborskii (Woloszinska) Seenayya et Subba-Raju, Geitlerinema amphibium (Agardh ex Gomont) Anagnostidis, Cyclotella meneghiniana Kützing and Synedra rumpens Kützing displayed higher biomasses in the dry season, whereas Microcystis aeruginosa (Kützing) Kützing, M. flos-aquae (Wittrock) Kirchner, Chlorella vulgaris Chodat and Cryptomonas ovata Ehrenberg displayed higher biomasses in the rainy season (Tab. 3).

C. raciborskii was the dominant species throughout the study (Tab. 3). In the dry season, this species had statistical differences as a function of depth at both sampling sites $\left(\mathrm{F}_{\mathrm{E} 1}=18.945\right.$ and $\mathrm{F}_{\mathrm{E} 2}=8.291 ; p<0.05$ and $p<0.01$, respectively), with higher biomass at the subsurface in $\mathrm{E}_{1}$ and near the sediment in $\mathrm{E}_{2}$. This species had higher biomass values in the dark hours in $\mathrm{E}_{1}$ and in the daylight hours in $\mathrm{E}_{2}$. In the rainy season, differences were only detected in $\mathrm{E}_{1}$ and $C$. raciborskii had a higher biomass at the subsurface, with an opposite light-dark pattern than that observed in the dry season.

S. rumpens was subdominant in the dry season, with biomasses higher than $5 \%$ of total biomass average in both sampling periods and seasons. C. meneghiniana contributed with $5 \%$ of the total biomass in $E_{1}$, particularly near the sediment. However, S. rumpens displayed significant horizontal differences, with the biomass enhanced in $\mathrm{E}_{2}(\mathrm{~F}=24.959, p<0.001)$. C. raciborskii was dominant in the rainy season, followed by $M$. aeruginosa, particularly at the subsurface in both seasons. Other taxa, such as M. flos-aquae, C. ovata and $S$. rumpens, were also important, with biomasses higher than $5 \%$ of total biomass average at least at one of the depths or sampling stations. M. flos-aquae and C. ovata were more abundant at the subsurface, with C. ovata having a more significant biomass in $\mathrm{E}_{2}$. Among subdominant species, only C. ovata and M. flos-aquae exhibited a light-dark pattern. C. ovata had higher biomass during daylight hours, particularly at the subsurface. M. flos-aquae had higher biomass during dark hours, except in $\mathrm{E}_{1}$ during the dry season.

The results from the Canonical Correspondence Analysis are displayed in Tab. 4 and 5 as well as Fig. 2 and 3. The Monte Carlo test proved significant $(p<0.01)$. There were relationships between environmental variables and biological information. 
Table 3. Phytoplanktonic species and average biomass ( $\left.\mu \mathrm{g} \cdot \mathrm{L}^{-1}\right)$ observed in the Mundaú reservoir, Northeastern Brazil. (-) dates without sampling.

\begin{tabular}{|c|c|c|c|c|c|c|c|c|c|c|c|c|c|c|c|c|}
\hline \multirow{3}{*}{ Species } & \multicolumn{4}{|c|}{$\mathrm{E}_{1}$ Dry season } & \multicolumn{4}{|c|}{$\mathrm{E}_{2}$ Dry season } & \multicolumn{4}{|c|}{$\mathrm{E}_{1}$ Rainy season } & \multicolumn{4}{|c|}{$\mathrm{E}_{2}$ Rainy season } \\
\hline & \multicolumn{2}{|c|}{$0.1 \mathrm{~m}$} & \multicolumn{2}{|c|}{$8 \mathrm{~m}$} & \multicolumn{2}{|c|}{$0.1 \mathrm{~m}$} & \multicolumn{2}{|c|}{$2 \mathrm{~m}$} & \multicolumn{2}{|c|}{$0.1 \mathrm{~m}$} & \multicolumn{2}{|c|}{$8 \mathrm{~m}$} & \multicolumn{2}{|c|}{$0.1 \mathrm{~m}$} & \multicolumn{2}{|c|}{$2 \mathrm{~m}$} \\
\hline & Light & Dark & Light & Dark & Light & Dark & Light & Dark & Light & Dark & Light & Dark & Light & Dark & Light & Dark \\
\hline \multicolumn{17}{|l|}{ BACILLARIOPHYTA } \\
\hline $\begin{array}{l}\text { Aulacoseira granulata (Ehrenberg) } \\
\text { Simonsen }\end{array}$ & - & 37 & - & - & - & 71 & - & - & - & - & - & - & - & - & - & - \\
\hline A. granulata var. angustissima Müller & 38 & 39 & - & 29 & 56 & - & 19 & - & - & 38 & - & - & - & - & - & 18 \\
\hline Cyclotella meneghiniana Kützing & 1886 & 1608 & 1304 & 1506 & 1435 & 1674 & 2094 & 1388 & 347 & 303 & 131 & 128 & 354 & 362 & 105 & 282 \\
\hline Diploneis ovalis (Hilse) Clève & - & 23 & - & 23 & - & - & 91 & - & - & - & - & - & - & - & - & - \\
\hline Eunotia major (Wm Smith) Rabenhorst & - & - & - & - & - & - & - & - & 249 & 19 & 20 & - & - & - & - & - \\
\hline E. monodon Ehrenberg & - & 75 & - & - & - & - & - & - & - & - & - & - & - & - & - & - \\
\hline Gomphonema augur Ehrenberg & - & - & - & - & - & - & - & 16 & - & - & 32 & - & - & - & - & - \\
\hline Navicula cuspidata Kützing & - & - & - & - & - & - & - & - & 19 & - & 32 & - & - & - & - & 22 \\
\hline Nitzschia palea (Kützing) W. Smith & - & 113 & 152 & 222 & 284 & 148 & 226 & 190 & 74 & - & 76 & - & - & - & - & 145 \\
\hline Pleurosigma sp. & - & - & - & - & - & - & - & - & 74 & - & - & - & - & - & - & - \\
\hline Synedra rumpens Kützing & 2680 & 2669 & 2433 & 2452 & 4357 & 4756 & 5266 & 4222 & 134 & 349 & 513 & 204 & 141 & 217 & 205 & 222 \\
\hline \multicolumn{17}{|l|}{ CHLOROPHYTA } \\
\hline Actinastrum hantzschii Lagerheim & - & - & 1 & - & 5 & - & - & - & - & 6 & 6 & - & 5 & 11 & - & 3 \\
\hline Ankistrodesmus gracilis (Reinsch) Korsikov & $v-$ & 1 & - & 1 & - & - & - & - & 4 & - & - & - & - & - & - & - \\
\hline $\begin{array}{l}\text { Botryococcus protuberans W. West \& } \\
\text { G.S. West }\end{array}$ & 428 & 147 & 587 & - & - & - & - & - & - & - & - & - & - & - & - & - \\
\hline Chlorella vulgaris Chodat & 63 & 69 & 41 & 58 & 677 & 502 & 342 & 185 & 406 & 492 & 474 & 400 & 457 & 261 & 289 & 256 \\
\hline $\begin{array}{l}\text { Closteriopsis acicularis (G.M. Smith) Belch } \\
\quad \& \text { Swale }\end{array}$ & - & 2 & - & - & 11 & - & 7 & - & - & - & - & - & - & - & - & - \\
\hline Closterium parvulum Nägeli & 201 & 152 & 168 & 176 & 195 & 147 & 228 & 141 & 75 & 50 & - & 75 & 72 & - & 48 & 100 \\
\hline Coelastrum microporum Nägeli & 87 & - & 15 & 15 & - & - & - & 30 & 29 & 29 & - & - & - & - & - & - \\
\hline C. pseudomicroporum Korsikov & 167 & 100 & 149 & 133 & - & 22 & 114 & 92 & 89 & 111 & 44 & 67 & 128 & 107 & 22 & 66 \\
\hline Crucigenia quadrata Morren & 67 & 58 & 10 & 35 & 6 & 6 & 77 & 42 & 51 & 51 & 56 & 12 & 18 & - & - & 7 \\
\hline Dictyosphaerium ehrenbergianum Nägeli & - & - & - & - & 18 & 36 & 5 & 9 & - & - & - & - & 4 & 9 & 4 & - \\
\hline D. pulchellum Wood & 13 & 28 & 24 & 17 & 30 & 15 & 41 & 40 & 15 & 10 & 20 & 18 & 12 & 7 & 11 & 6 \\
\hline Golenkinia radiata Chodat & - & - & - & - & - & - & - & - & - & - & - & - & - & - & - & - \\
\hline Kirchneriella lunaris (Kirchn.) Möebius & 2 & 1 & - & 1 & 11 & 11 & 4 & 1 & 6 & 9 & 1 & 1 & 4 & 2 & 5 & 3 \\
\hline K. obesa $($ W. West) Schmidle & 15 & 14 & 6 & 9 & 13 & 21 & 8 & 11 & 6 & 7 & 2 & 2 & 9 & 9 & 4 & 7 \\
\hline $\begin{array}{l}\text { Monoraphidium arcuatum (Korsikov) } \\
\text { Hindák }\end{array}$ & 11 & 6 & 6 & 14 & 43 & 50 & 29 & 10 & 21 & 71 & 28 & 35 & 49 & 39 & 52 & 56 \\
\hline M. circinale (Nygaard) Nygaard & 3 & 4 & 4 & 4 & 1 & 1 & 2 & 3 & - & - & - & - & - & - & - & - \\
\hline $\begin{array}{l}\text { M. contortum } \text { (Thuret) } \\
\text { Komárkova-Legnerová }\end{array}$ & 34 & 23 & 71 & 62 & 24 & 27 & 58 & 67 & 19 & 26 & 6 & 7 & 14 & 18 & 21 & 19 \\
\hline M. griffithi (Berkel) Komárkova-Legnerová & á 7 & 4 & 3 & 6 & 2 & 6 & 15 & 14 & 24 & 18 & 7 & 15 & 13 & 7 & 10 & 10 \\
\hline M. pusillum (Printz) Komárkova-Legnerová & rá 14 & 14 & 25 & 26 & 12 & 23 & 27 & 31 & 10 & 11 & 5 & 3 & 10 & 3 & 6 & $\begin{array}{c}9 \\
\text { continua }\end{array}$ \\
\hline
\end{tabular}




\begin{tabular}{|c|c|c|c|c|c|c|c|c|c|c|c|c|c|c|c|c|}
\hline \multirow{3}{*}{ Species } & \multicolumn{4}{|c|}{$\mathrm{E}_{1}$ Dry season } & \multicolumn{4}{|c|}{$\mathrm{E}_{2}$ Dry season } & \multicolumn{4}{|c|}{$\mathrm{E}_{1}$ Rainy season } & \multicolumn{4}{|c|}{$\mathrm{E}_{2}$ Rainy season } \\
\hline & \multicolumn{2}{|c|}{$0.1 \mathrm{~m}$} & \multicolumn{2}{|c|}{$8 \mathrm{~m}$} & \multicolumn{2}{|c|}{$0.1 \mathrm{~m}$} & \multicolumn{2}{|c|}{$2 \mathrm{~m}$} & \multicolumn{2}{|c|}{$0.1 \mathrm{~m}$} & \multicolumn{2}{|c|}{$8 \mathrm{~m}$} & \multicolumn{2}{|c|}{$0.1 \mathrm{~m}$} & \multicolumn{2}{|c|}{$2 \mathrm{~m}$} \\
\hline & Light & Dark & Light & Dark & Light & Dark & Light & Dark & Light & Dark & Light & Dark & Light & Dark & Light & Dark \\
\hline Oocystis lacustris Chodat & - & - & - & - & 1 & 3 & 5 & - & - & - & 57 & 10 & - & - & - & - \\
\hline Pediastrum tetras (Ehrenberg) A. Braun & - & - & - & - & - & - & - & - & - & - & - & - & - & - & - & - \\
\hline $\begin{array}{l}\text { Scenedesmus acuminatus (Langeheim) } \\
\text { Chodat }\end{array}$ & 15 & - & 4 & - & - & 7 & - & 8 & 15 & 8 & 8 & 30 & 22 & - & - & 15 \\
\hline $\begin{array}{l}\text { S. acuminatus var. bernardii (G. M. Smith) } \\
\text { Dedussenko }\end{array}$ & 1) & - & - & - & - & - & 3 & - & 3 & 8 & - & 3 & 5 & 3 & - & 3 \\
\hline S. acutus Meyen & - & - & - & - & - & - & - & - & - & - & - & - & - & - & - & 8 \\
\hline S. arcuatus Lemmermann & 1 & 3 & - & - & - & - & - & 7 & 6 & 3 & - & - & - & - & - & - \\
\hline S. bernardii G. M. Smith & - & - & 5 & - & - & - & - & 9 & 9 & - & - & - & - & 9 & 9 & - \\
\hline S. bicaudatus (Hansgirg) Chodat & - & - & 5 & 3 & - & - & 4 & 9 & - & - & - & - & - & - & - & 2 \\
\hline S. ecornis (Ehrenberg) Chodat & - & 2 & 2 & 2 & - & - & 3 & - & - & - & 2 & - & 3 & - & - & - \\
\hline S. quadricauda (Turpin) Brébisson & 7 & 7 & 16 & 4 & 13 & 48 & 14 & 21 & 18 & 31 & 14 & 9 & 43 & 17 & 17 & 9 \\
\hline S. quadricauda var. parvus G. M. Smith & 48 & 26 & 33 & 28 & 18 & 22 & 11 & 38 & 4 & 11 & 2 & 2 & 2 & - & 6 & 4 \\
\hline Scenedesmus sp. & - & - & - & - & - & - & - & 4 & - & 18 & - & - & - & 4 & - & - \\
\hline Tetraedron caudatum (Corda) Hansgirg & - & - & - & 1 & - & - & - & 4 & - & - & - & - & - & - & - & - \\
\hline T. gracile (Reinsch) Hansgirg & 38 & - & - & - & - & - & - & - & - & - & - & - & - & - & - & - \\
\hline T. incus (Teiling) G. M. Smith & 10 & 12 & 14 & 17 & 24 & 3 & 7 & 10 & - & 3 & - & - & - & - & - & - \\
\hline T. mediocris Hindák & - & 2 & - & - & - & - & 2 & 2 & - & - & - & - & - & - & - & - \\
\hline T. minimum (A. Braun) Hansgirg & 16 & 35 & 65 & 51 & 24 & 20 & 65 & 35 & 28 & 24 & 4 & 8 & 4 & 16 & 8 & - \\
\hline T. triangulare (Chodat) Komárek & 35 & 39 & 25 & 26 & 27 & 12 & 25 & 23 & 15 & 12 & 6 & 3 & 12 & 6 & 18 & 6 \\
\hline T. victoriae Wolosynska & - & - & - & - & - & - & - & - & - & 73 & - & - & - & - & - & - \\
\hline Tetrastrum elegans Playfair & 6 & 3 & 55 & 35 & 18 & 6 & 26 & 16 & 13 & 19 & 6 & 31 & - & 6 & 6 & 13 \\
\hline \multicolumn{17}{|l|}{ CYANOPHYTA } \\
\hline Chroococcus limneticus Lemmermann & - & - & - & - & 73 & 42 & 37 & 3 & 26 & 21 & 16 & 16 & 15 & 16 & 26 & 32 \\
\hline C. minutus Kützing & 28 & 33 & 21 & 30 & 10 & 12 & 18 & 17 & 11 & 10 & 9 & 11 & 20 & 6 & 10 & 4 \\
\hline C. turgidus Kützing & 33 & 62 & 21 & 24 & 104 & 106 & 64 & 22 & 51 & 85 & 85 & 61 & 313 & 153 & 184 & 80 \\
\hline $\begin{array}{l}\text { Cylindrospermopsis raciborskii (Wolz.) } \\
\text { Seenayya et Subba-Raju }\end{array}$ & 53238 & 55465 & 23438 & 29849 & 41018 & 38808 & 59439 & 49189 & 31242 & 25563 & 9310 & 11868 & 18380 & 19014 & 18382 & 19626 \\
\hline $\begin{array}{l}\text { Geitlerinema amphibium (Agardh ex } \\
\text { Gomont) Anagnostidis }\end{array}$ & 550 & 642 & 1350 & 939 & 323 & 361 & 805 & 874 & 578 & 541 & 394 & 333 & 409 & 386 & 438 & 574 \\
\hline Merismopedia minima Beck & 17 & 13 & 41 & 38 & 5 & 2 & 13 & 19 & 7 & 6 & 10 & 1 & 4 & 2 & 1 & 3 \\
\hline M. punctata Meyen & 263 & 250 & 251 & 242 & 84 & 182 & 240 & 281 & 112 & 104 & 297 & 379 & 79 & 38 & 80 & 91 \\
\hline Microcystis aeruginosa (Kützing) Kützing & 1661 & 795 & 831 & 741 & 129 & 221 & 775 & 1553 & 1257 & 1571 & 398 & 788 & 1422 & 1309 & 2104 & 1031 \\
\hline M. flos-aquae (Wittrock) Kirchner & 848 & 125 & 187 & 61 & - & 242 & 612 & 550 & 848 & 1937 & 241 & 363 & 232 & 838 & 843 & 729 \\
\hline M. panniformis Komárek et al. & - & - & - & - & - & - & - & 39 & - & - & - & - & - & - & - & - \\
\hline M. wesenbergii (Komárek) Komárek & - & - & - & - & - & - & - & - & - & 190 & 98 & - & 182 & 95 & 91 & - \\
\hline Pseudanabaena catenata Lauterborn & 268 & 149 & 8 & 8 & 15 & 7 & 85 & 19 & 53 & 68 & - & - & 29 & 15 & 67 & 31 \\
\hline Raphidiopsis mediterranea Skuja & 1606 & 1042 & 103 & 286 & 459 & 406 & 1113 & 1384 & 1014 & 928 & 186 & 334 & 754 & 487 & 714 & 813 \\
\hline Spirulina sp. & - & - & - & - & - & - & - & - & - & - & - & - & - & - & - & - \\
\hline
\end{tabular}




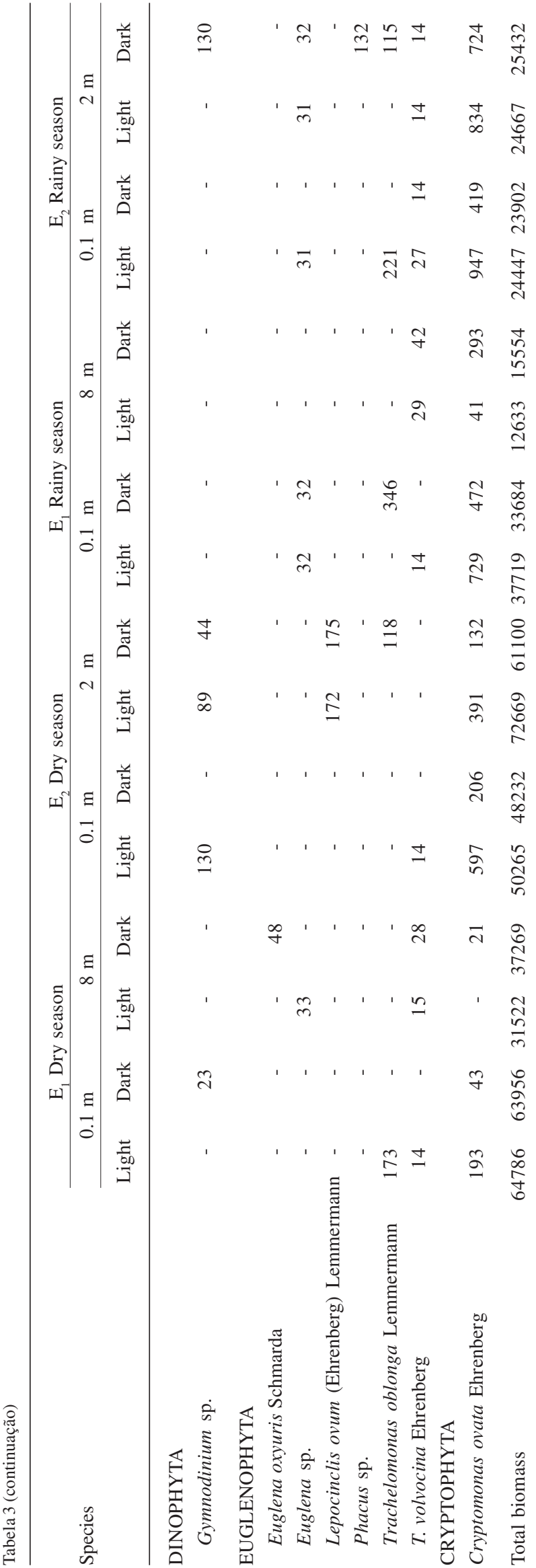

Table 4. Statistical synthesis of the CCA first two axes performed in the Mundaú reservoir Northeastern Brazil.

\begin{tabular}{lrr}
\hline & Axis 1 & Axis 2 \\
\hline Eigenvalue & 0.062 & 0.015 \\
Variance percentage explained & 22.0 & 5.4 \\
Variance percentage accumulated & 22.0 & 27.4 \\
Pearson correlation (species-environment) & 0.934 & 0.844 \\
Monte Carlo test & & \\
$\quad$ Eingenvalues $-p$ & 0.01 & 0.03 \\
$\quad$ Correlations species-environment & 0.01 & 0.01 \\
\hline
\end{tabular}

Eigenvalues of Axis 1 and 2 explain $27.4 \%$ of the biological data variance. The correlation of the species with environmental conditions was high on both axes.

CCA Axis 1 clearly split the seasons. The sampling units in the dry period were positively related to the axis, whereas those in the rainy period were negatively related. Taking into account the intra-set correlation, the variables positively associated to Axis 1 were temperature, $\mathrm{pH}$, total dissolved phosphorus and orthophosphate, and a grouping of sampling units in the dry season was observed. These environmental variables had higher values in this season (Tab. 1 and 2). The chlorophyta Closterium parvulum Nägeli, Monoraphidium contortum (Thret) Komárkova-Legnerová and Scenedesmus quadricauda var. parvus G.M. Smith, cyanobacteria such as Cylindrospermopsis raciborskii and the diatoms Aulacoseira granulata (Ehrenberg) Simonsen, Cyclotella meneghiniana and Synedra rumpens all displayed higher biomasses in the dry season (Tab. 3).

Dissolved oxygen, electrical conductivity, total nitrogen and nitrite were negatively associated to Axis 1 and a grouping of sampling units in the rainy period was

Table 5. Canonic coefficients and intra-set correlation of the environmental variables for the axis 1 and 2 in the Mundaú reservoir Northeastern Brazil.

\begin{tabular}{lrrrr}
\hline & \multicolumn{2}{c}{$\begin{array}{c}\text { Canonical } \\
\text { coefficient }\end{array}$} & \multicolumn{2}{c}{$\begin{array}{c}\text { Correlation } \\
\text { coefficient } \\
\text { (intra-set) }\end{array}$} \\
\cline { 2 - 5 } & Axis 1 & Axis 2 & Axis 1 & Axis 2 \\
\hline Temperature & 0.208 & -1.396 & 0.884 & -0.320 \\
Dissolved oxygen & -0.071 & -0.376 & -0.810 & -0.313 \\
Electric conductivity & -0.339 & 0.601 & -0.964 & 0.018 \\
Turbidity & -0.154 & -1.356 & -0.490 & -0.163 \\
pH & 0.050 & 1.172 & 0.626 & -0.411 \\
Total nitrogen & -0.087 & 0.731 & -0.769 & 0.299 \\
Nitrate & -0.086 & -0.230 & -0.490 & 0.344 \\
Nitrite & 0.108 & 0.008 & -0.706 & 0.095 \\
Total phosphorus & 0.182 & 1.282 & 0.279 & 0.411 \\
Total dissolved phosphorus & 0.308 & 0.394 & 0.902 & 0.059 \\
Orthophosphate & -0.051 & -0.197 & 0.738 & 0.031 \\
\end{tabular}


observed. These environmental variables had higher values in this period (Tab. 1 and 2). The Chlorophyta Chlorela vulgaris, Monoraphidium arcuatum (Korsikov) Hindák and M. griffithii (Berkel) Komárkova-Legnerová, the cyanobacteria Chroococcus turgidus Kützing, Microcystis aeruginosa and M. flos-aquae, and phytoflagellates such as Cryptomonas ovata and Trachelomonas volvocina Ehrenberg displayed higher biomasses in the rainy period (Tab. 3).

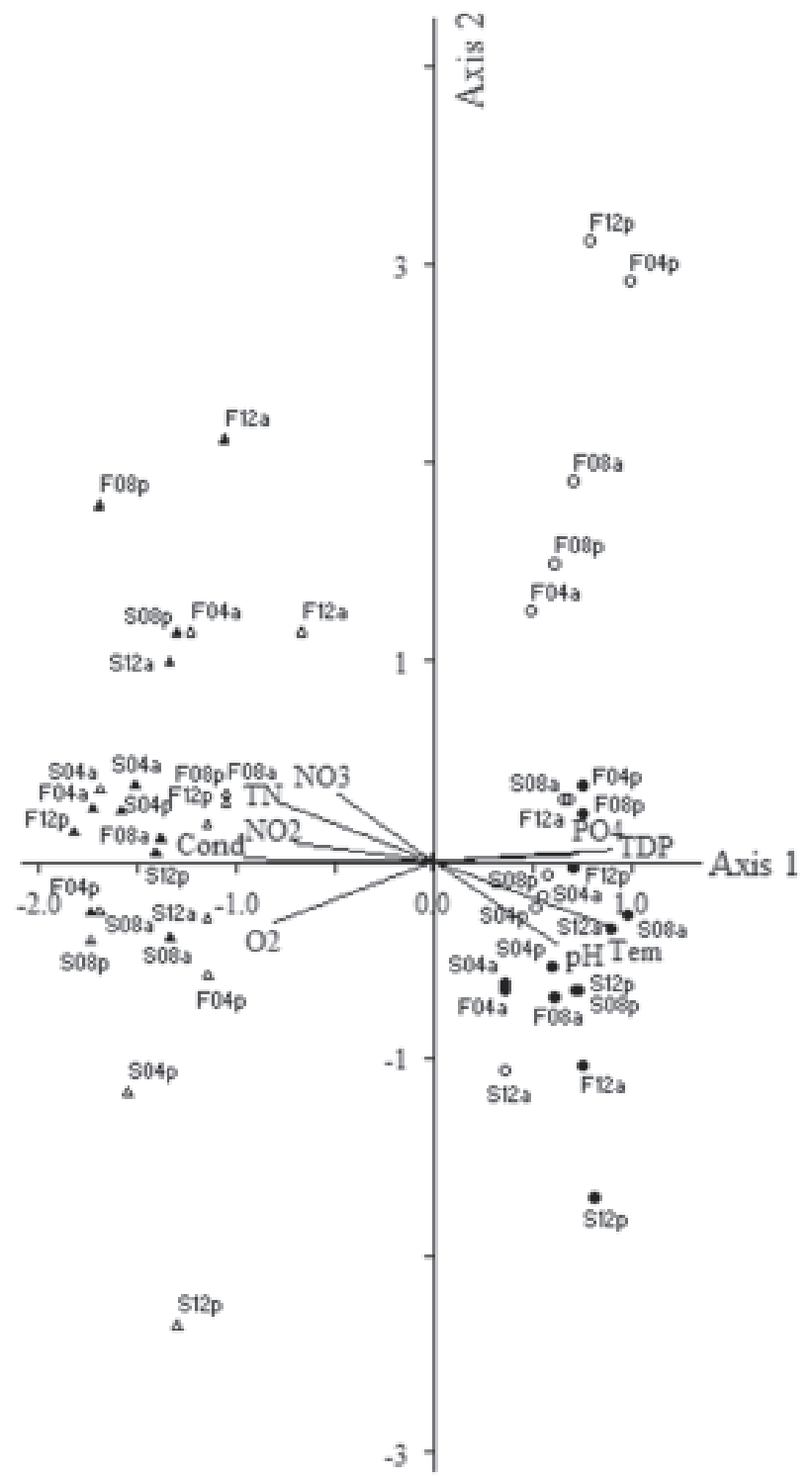

Figure 2. CCA ordination of the sampling unities, on the basis of the environmental variables of the Mundaú reservoir, Northeastern Brazil. The unities were identified according with the sampling station and the season period. $\bigcirc=\mathrm{E}_{1}$ Dry; $\bullet=\mathrm{E}_{2}$ Dry; $\triangle=\mathrm{E}_{1}$ Rainy; $\boldsymbol{\Delta}=\mathrm{E}_{2}$ Rainy. The unities identification respectively corresponds to the depths ( $\mathrm{S}=$ Subsurface; $\mathrm{B}=$ Bottom) and daylight ( $8 \mathrm{am}, 12 \mathrm{pm}$ and $4 \mathrm{pm})$ and dark ( $8 \mathrm{pm}, 12$ am and 4 am) hours. Abbreviations: Cond = Electrical conductivity $; \mathrm{NO}_{2}=$ Nitrite $; \mathrm{NO}_{3}=$ Nitrate $\mathrm{O}_{2}=$ Dissolved oxygen $; \mathrm{pH}=\mathrm{pH} ; \mathrm{PO}_{4}=$ Orthophosphate; Tem $=$ Temperature; $\mathrm{TN}=$ Total nitrogen; $\mathrm{TDP}=$ Total dissolved phosphorus; $\mathrm{TP}=$ Total phosphorus; Tur $=$ Turbidity.
Spatial and temporal variations (between hours) were observed on Axis 2. Spatial differences between sampling stations were more pronounced in the dry season, whereas differences between the light and dark hours were more pronounced in the rainy period. The variables positively associated to Axis 2 were $\mathrm{pH}$ and total phosphorus, whereas turbidity and temperature were negatively associated to the axis. In the dry season, $\mathrm{E}_{1}$ exhibited vertical differences and the CCA Axis 2 separated the sampling units from the subsurface and bottom, which were negatively and positively associated to the axis, respectively. Vertical differences were not observed in $\mathrm{E}_{2}$ and all sampling units from this station were grouped in the CCA and negatively associated to Axis 2 . In the dry period, the algae with higher biomasses in $\mathrm{E}_{1}$ were mostly the cyanobacteria Chroococcus minutus Kützing, C. raciborskii, Geitlerinema amphibium and Pseudanabaena catenata Lauterborn. Of these, only G. amphibium had higher biomasses at the bottom. The algae in $\mathrm{E}_{2}$ with higher biomasses were the Chlorophyta C. vulgaris, M. arcuatum and Scenedesmus quadricauda (Turpin) Brébisson, the cyanobacteria Chroococcus limneticus Lemmermann and C. turgidus, diatoms such as $S$. rumpens, and phytoflagellates such as $C$. ovata (Tab. 3).

CCA Axis 2 for the rainy season separated the sampling units from the light and dark hours, which were negatively and positively associated to the axis, respectively. The algae in this period with higher biomasses during daylight hours were the Chlorophyta Dictyosphaerium pulchellum Wood, Scenedesmus quadricauda and Tetraedron triangulare (Chodat) Komárek and phytoflagellates such as Cryptomonas ovata. Cyanobacteria such as Microcystis flos-aquae and the Chlorophyta Coelastrum pseudomicroporum Korsikov, Monoraphidium arcuatum, M. contortum, and Tetrastrum elegans Playfair had higher biomasses during dark hours (Tab. 3).

\section{Discussion}

Eutrophication is a frequent environmental problem in bodies of water throughout the world. Negative impacts include the excessive growth of certain groups of organisms, such as blue-green algae. Cyanobacteria are favored by a wide range of environmental factors. While they can occur in oligotrophic and mesotrophic systems (Hecky \& Kling 1987; Huszar \& Caraco 1998), these cyanobacteria are prevalent under eutrophic conditions, thriving in warm water temperatures (Shapiro 1990), low luminosity (Padisák \& Reynolds 1998), alkaline pH (Reynolds \& Walsby 1975) and high nutrient concentrations, 
particularly phosphorus (Watson et al. 1997) and low $\mathrm{N}: \mathrm{P}$ ratio (Smith 1983). Such conditions are found in the Mundaú reservoir and explain the development of substantial cyanobacteria biomass in this system.

Nostocales, Oscillatoriales and Chroococcales all occur in the Mundaú reservoir. Nostocales species generally require a considerable luminosity and high temperatures. They are also more resistant to nutrient depletion, particularity nitrogen (Dokuil \& Teubner 2000). Oscillatoriales are typical of high turbidity environments and low luminosity conditions (Reynolds 1997). Selfshadowing, due to the considerable development of

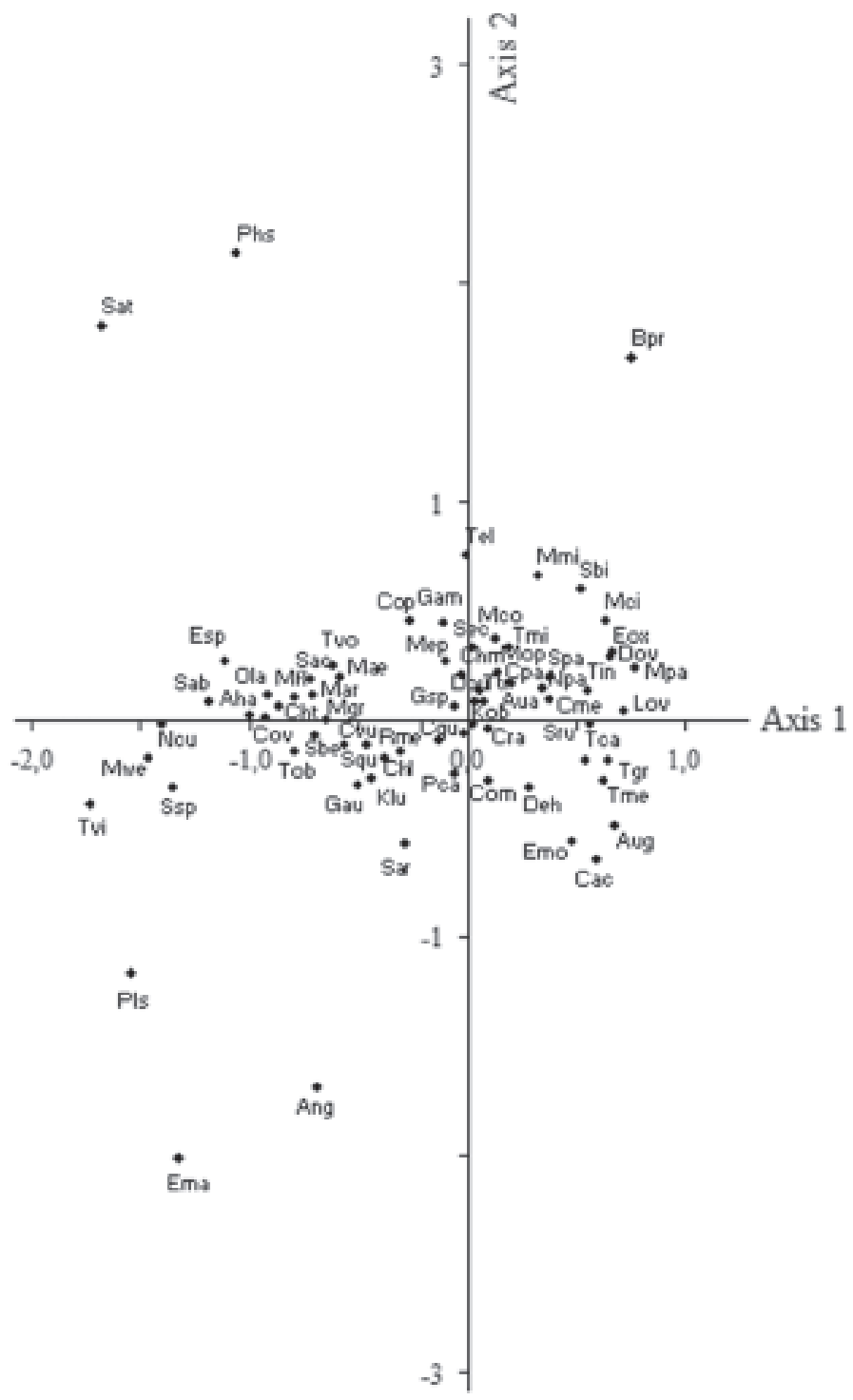

Figure 3. CCA ordination of the phytoplanktonic species found in the Mundaú reservoir, Northeastern Brazil. Abbreviations: Aha = Actinastrum hantzschii $;$ Ang = Ankistrodesmus gracilis $;$ Aug = Aulacoseira granulata $;$ Aua = A. granulata var. angustissima $; \mathrm{Bpr}=\mathrm{Botryococcus} \mathrm{protuberans;}$ Cac $=$ Closteriopsis acicularis $; \mathrm{Chl}=$ Chroococcus limneticus $; \mathrm{Chm}=$ C. minutus $; \mathrm{Cht}=$ C. turgidus $;$ Cme = Cyclotella meneghiniana; Com = Coelastrum microporum $;$ Cop =C. pseudomicroporum $;$ Cov = Cryptomonas ovata $;$ Cpa = Closterium parvulum Cqu $=$ Crucigenia quadrata $; \mathrm{Cra}=$ Cylindrospermopsis raciborskii $; \mathrm{Cvu}=$ Chlorella vulgaris $;$ Deh $=$ Dictyosphaerium ehrenbergianum; Dpu $=$ D. pulchellum; Dov $=$ Diploneis ovalis $;$ Ema $=$ Eunotia major $;$ Emo = E. monodon $;$ Eox $=$ Euglena oxyuris $;$ Esp = Euglena sp $;$ Gam = Geitlerinema amphibium; $\mathrm{Gau}=$ Gomphonema augur $; \mathrm{Gra}=$ Golenkinia radiata $; \mathrm{Klu}=$ Kirchneriella lunaris $; \mathrm{Kob}=$ K. obesa $;$ Lov $=$ Lepocinclis ovum $;$ Mae $=$ Microcystis aeruginosa $;$ Mar = Monoraphidium arcuatum $;$ Mci $=$ M. circinale $;$ Mco = M. contortum $;$ Mep = Merismopedia punctata $;$ Mfl = Microcystis flos-aquae; $\mathrm{Mgr}=$ Monoraphidium griffithii $; \mathrm{MMi}=$ Merismopedia minima $;$ Mop = Monoraphidium pusillum; Mpa = Microcystis panniformis; Mwe = M. wesenbergii $; \mathrm{Ncu}=$ Navicula cuspidata $; \mathrm{Npa}=$ Nitzschia palea $; \mathrm{Ola}=$ Oocystis lacustris $; \mathrm{Pca}=$ Pseudanabaena catenata $;$ Phs $=$ Phacus sp.; Pls = Pleurosigma sp.; Pte = Pediastrum tetras $; \mathrm{Rme}=$ Raphidiopsis mediterranea $; \mathrm{Sab}=$ Scenedesmus acuminatus var. bernardii $;$ $\mathrm{Sac}=S$. acuminatus $; \mathrm{Sar}=S$. arcuatus $; \mathrm{Sat}=S$. acutus $; \mathrm{Sbe}=S$. bernardii $; \mathrm{Sbi}=S$. bicaudatus $; \mathrm{Sec}=$ S. ecornis $;$ Squ $=S$. quadricauda $;$ $\mathrm{Spa}=$ S. quadricauda var. parvus $; \mathrm{Sps}=$ Spirulina $\mathrm{sp} . ;$ Sru = Synedra rumpens; $\mathrm{Ssp}=$ Scenedesmus $\mathrm{sp} . ; \mathrm{Tca}=$ Tetraedron caudatum $;$ Tel $=$ Tetrastrum elegans $;$ Tgr $=$ Tetraedron gracile $;$ Tin $=T$. incus $;$ Tme $=T$. mediocris $;$ Tmi $=T$. minima $;$ Tob $=$ Trachelomonas oblonga $;$ Ttr $=$ Tetraedron triangulare $; \mathrm{Tvi}=$ T. victoriae $;$ Tvo $=$ Trachelomonas volvocina . 
heterocytes, results in a decreased availability of luminosity, thereby accelerating the succession process. The Nostocales nitrogen pool is then released for the Oscillatoriales by means of cell membrane disruption.

The above-mentioned conditions enable the occurrence of a considerable development of species that are able to fix nitrogen and tolerate low luminosity (Pádisák \& Reynolds 1998; Dokuil \& Teubner 2000). Cylindrospermopsis raciborskii has been reported as an opportunistic species (Istvánovics et al. 2000) able to maintain perennial populations, particularly in tropical systems (Briand et al. 2002). Due to its similarity to the ecology of Oscillatoriales, Pádisak \& Reynolds (1998) moved this species to a new assemblage (denominated $\mathrm{Sn}$ ), characterized by living in hot, de-stratified systems, while capable of tolerating low luminosity and low nitrogen concentrations. The strategies facilitating dominance of this taxa include: 1) proliferation at high temperatures; 2) first choosing $\mathrm{NH}_{3}$ and $\mathrm{NO}_{3}$ (Saker et al. 1999); 3) affinity and stocking of phosphorus (Istvánovics et al. 2000); 4) frequently reported as herbivore resistant (Fabbro \& Duivenvoorden 1996); and $5)$ the ability to regulate its position in the water column, mostly during thermally stratified periods (Pádisak 1997). We may therefore consider this species an R-strategist, which has developed under the favorable conditions in the reservoir investigated.

The most representative colony-forming taxa of cyanobacteria in the Mundaú reservoir belong to the Microcystis genus. The blooming formation of species from this genus is related to the occurrence of anoxia near the sediment (Reynolds 1984). Sediment anoxia is responsible for the non-stop release of phosphorus from the sediment, which favors the maintenance of Microcystis blooms at the subsurface of the system (Calijuri \& Dos Santos 1996). Microcystis dominance in tropical systems may be favored by changes in the mixture throughout the day (Reynolds et al. 1987). Microcystis species are well known to be generally highly toxic algae, responsible for a number of public health problems. The most worldwide-known incidents took place in the northeastern region of Brazil, where Microcystis blooms killed fish and shrimp in the state of Rio Grande do Norte (Chellappa et al. 2000) and led to death over 70 patients in a dialysis clinic in Caruaru, Pernambuco (Jochimsen et al. 1998). However, blooms of species from the Microcystis genus were massively replaced by Cylindrospermopsis raciborskii, particularly in the southeastern region of Brazil, as observed in the Juturnaíba reservoir, Rio de Janeiro (Marinho \& Huszar 2002) and the Garças reservoir in São Paulo (Tucci \& Sant'Anna 2003). This species has been well documented as the cause of blooms in the northeastern region (Bouvy et al. 2000; Huszar et al. 2000), including the Mundaú reservoir (Moura et al. 2007).

Chlorophyta were represented particularly by Chlorococcales, which are generally small in size while having a high surface/volume ratio. This favors the nutrient absorption, thereby allowing adaptive advantages, especially in oligotrophic waters (Negro et al. 2000). Based on the phytosociological assemblages proposed by Reynolds (1997), the green algae found in the Mundaú reservoir, such as Chlorella vulgaris and Monoraphidium contortum, are grouped within the $\mathrm{X}_{1}$ assemblages. Therefore, they are found in shallow, mixed, stratified environments and are not sensitive to nutrient shortages.

The importance of Bacillariophyta in eutrophic systems is enhanced by changes in nutrient availability and water transparency (Sommer 1988). S. rumpens and C. meneghiniana (the taxa present in the reservoir investigated) are typical of shallow, turbid, enriched systems (especially S. rumpens), but are generally sensitive to nutrient depletion. Sommer (1988) states that diatoms are excellent competitors for reactive soluble phosphorus and this could explain the occurrence of higher densities of these algae in the dry seasons. Tilman \& Kilham (1976) found that Cyclotella meneghiniana has a greater dependency on phosphorus when compared to silicates. This corroborates our observations in the Mundaú reservoir, particularly in the dry season.

Cryptomonas ovata had the highest greater number of individuals among the flagellates. This species belongs to the Y functional group (Reynolds 1997), which is usually found in eutrophic systems and is resistant to poor luminosity. A greater biomass of this taxon in the reservoir investigated occurred in the rainy season, when thermal stratification is absent. This finding is in agreement with Romo \& Miracle (1995), who observed that the development of Cryptomonas populations come about immediately following the occurrence of rains, as rain is responsible for the circulation of water throughout the water column and the re-suspension of nutrients trapped in the sediment.

The Canonical Correspondence Analysis revealed that the development of the phytoplankton community in the dry season was mainly influenced by the availability of phosphorus. The positive correlations between biotic variables and phosphorus products strongly suggest that bottom-up effects play a role in phytoplankton biomass control.

In the dry season, measurements of water temperature, dissolved oxygen, turbidity and $\mathrm{pH}$ exhibited differences as a function of depth, with higher values found at the subsurface. These variables influence the spatial distribution of the phytoplankton community. Cyanobacteria, which are dominant in the pelagic zone, 
had biomasses with significant vertical differences between depths, with higher values at the subsurface, particularly for Cylindrospermopsis raciborskii. Although the dominance of $C$. raciborskii persisted in the littoral zone, a higher abundance of diatoms was observed, particularly Synedra rumpens and cocoid chlorophyta.

The algae biomass decrease in the rainy season was driven by events associated to thermal de-stratification and chemical stratification, especially dissolved oxygen and nitrogen. Phytoplankton development was influenced by an increase in allochtonous particulate nutrients, which gave rise to community reorganization events, thereby contributing to the establishment of C. raciborskii and species from the Microcystis genus.

Water temperature and phosphorus were generally the abiotic variables with a greater influence over both spatial distribution (pelagic/littoral zones and depths) and temporal distributions (times of the day and seasons) of the phytoplankton community in the Mundaú reservoir in northeastern Brazil. In the present study, the demineralization of phosphorus during the dry season contributed toward the re-suspension of diatom frustules. In the rainy season, the circulation of the water column and increase in particulate elements, which were responsible for the low availability of resources, explained the development of the large biomass of Cryptomonas ovata and Microcystis species. C. raciborskii was the species that most stood out in the Mundaú reservoir for being predominant in both seasons. The establishment of cyanobacteria blooms, mainly made up of C. raciborskii, was more pronounced in the dry season, which certainly occurred due to the high availability of phosphorus, low $\mathrm{NO}_{3}$ levels, limited nitrogen, alkaline $\mathrm{pH}$ and thermal stability of the system.

\section{Acknowledgements}

We are grateful for the grants from Programa de Pós Graduação em Botânica (PPGB) of the Universidade Federal Rural de Pernambuco (UFRPE) and from the Conselho Nacional de Desenvolvimento Científico (CNPq), Processes 130109/2004-5, 503850/2003-9 as well as the grants to ANM (300612/2005-20).

\section{References}

Ayres, M.; Ayres Júnior, M.; Ayres, D.L. \& Dos Santos, A.A. 2003. Software BioEstat, aplicações estatísticas nas áreas das ciências biomédicas. Versão 3.0. Belém, Sociedade Civil Mamirauá/ MCT/ CNPq.

Bouvy, M.; Molica, R.; De Oliveira, S.; Marinho, M. \& Becker, B. 1999. Dynamics of a toxic cyanobacterial bloom (Cylindrospermopsis raciborskii) in a shallow reservoir in the semi-arid region of northeast Brazil. Aquatic Microbial Ecology 20: 285-297.
Bouvy, M.; Falcão, D.; Marinho, M.; Pagano, M. \& Moura, A. 2000. Occurrence of Cylindrospermopsis (Cyanobacteria) in 39 brazilian tropical reservoirs during the 1998 drought. Aquatic Microbial Ecology 23: 13-27.

Bouvy, M.; Molica, R.; Oliveira, S.; Marinho, M. \& Beker, B. 2001. Effects of a cyanobacterial bloom (Cylindrospermopsis raciborskii) on bacterial and zooplankton communities in Ingazeira reservoir (Northeast, Brazil). Aquatic Microbial Ecology 25: 215-227.

Briand, J.F.; Robillot, C.; Quiblier-Llobéras, C.; Humbert, J.F.; Couté, A. \& Bernard, C. 2002. Environmental context of Cylindrospermopsis raciborskii (Cyanobacteria) blooms in a shallow pond in France. Water Research 36: 3183-3192.

Briand, J.F.; Lebourlanger, C.; Humbert, J.; Bernard, C. \& Dufour, P. 2004. Cylindrospermopsis raciborskii (Cyanobacteria) invasion at mid-latitudes: selection, wide physiological tolerance, or global warming? Journal of Phycology 40: 231-238.

Calijuri, M.C. \& Dos Santos, A.C.A. 1996. Short-term changes in the Barra Bonita reservoir (São Paulo, Brazil): emphasis on the phytoplankton communities. Hydrobiologia 330: 163-175.

Chellappa, N.T. \& Costa, M.A.M. 2003. Dominant and co-existing epecies of Cyanobacteria from a eutrophicated reservoir of Rio Grande do Norte State, Brazil. Acta Oecologica 24: S3-S10.

Chellappa, N.T.; Costa, M.A.M. \& Marinho, I.R. 2000. Harmful cyanobacterial blooms from semiarid freshwater ecosystems of North-East Brazil. Australian Society of Limnology 38: 45-49.

Dokuil, M.T. \& Teubner, K. 2000. Cyanobacterial dominance in lakes. Hydrobiologia 438: 1-12.

Edler, L. 1979. Recommendations for marine biological studies in the Baltic Sea: phytoplankton and chlorophyll. The Baltic Marine Biologists 5: 1-38.

Fabbro, L.D. \& Duivenvoorden, L.J. 1996. Profile of a bloom of the cyanobacteria Cylindrospermopsis raciborskii (Wolozysnska) Seenaya and Subba Raju in the Fitzroy River in tropical center Queensland. Marine and Freshwater Research 47: 685-694.

Golterman, H.; Clymo, R.S. \& Ohnstad, M.A.M. 1971. Methods for physical and chemical analysis of freshwaters. $2^{\text {nd }} \mathrm{ed}$, Oxford, Blackwell Scientific Publications.

Hasle, G.R. 1978. The inverted-microscope methods. Pp. 88-96. In: A. Sournia (ed.). Phytoplankton manual. Paris, UNESCO.

Hecky, R. \& Kling, H.J. 1987. Phytoplankton ecology of the great lakes in the rift valleys of central Africa. Archiv für Hydrobiologie Beihefte Ergebnisse der Limnologie 25: 197-228.

Huszar, V.L.M. \& Caraco, N.F. 1998. The relationship between phytoplankton composition and physical-chemical variables: a comparison of taxonomic and morphological-functional descriptors in six temperate lakes. Freshwater Biology 40: 679-696.

Huszar, V.L.M. \& Reynolds, C.S. 1997. Flood-plain lake (Lago Batata, Pará, Brazil): responses to gradual environmental change. Hydrobiologia 346: 169-181.

Huszar, V.L.M.; Silva, L.H.S.; Marinho, M.; Domingos, P. \& Sant'Anna, C.L. 2000. Cyanoprokaryote assemblages in eight productive tropical Brazilian waters. Hydrobiologia 424: 67-77.

Istvánovics, V.; Shafik, H.M.; Préssing, M. \& Juhos, S. 2000. Growth and phosphate uptake kinetics of the cyanobacterium Cylindrospermopsis raciborskii (Cyanophyceae) in a throughflow cultures. Freshwater Biology 43: 257-275.

Jochimsen, E.M.; Carmichael, W.W.J.; Cardo, D.M.; Cookson, S.T.; Holmes, C.E.M.; Antunes, B.C.; Melo Filho, D.A.; Lyra, T.M.; Barreto, V.S.T.; Azevedo, S.M.F.O. \& Jarvis, W.R. 1998. Liver failure and death after exposure to microcystins at a hemodialysis center in Brazil. New England Journal Medicine 338: 873-878.

Komárek, J. \& Foot, B. 1983. Das Phytoplankton des Sübwassers Systematik und Biologie. 7. Teil: Chlorophyceae (Grünalgen) Ordnung: Chlorococcales. Pp. 1-1044. In: H.J. Elster \& W. Ohle (eds.). Die Binnengewässer. Stutgart, Begründet von August Thienemann. 
Komárek, J. \& Anagnostidis, K. 1999. Cyanoprokaryota. 1. Teil: Chroococcales. In: H. Ettl; G. Gärtner; H. Heynig \& D. Mollenhauer (eds.). Süsswasserflora von Mitteleuropa. Stutgart, Gustav Fischer.

Komárek, J. \& Anagnostidis, K. 2005. Cyanoprokaryota 2. Teil: Oscillatoriales. Pp. 1-759. In: B. Bridel; G.L. Gastner \& M.S. Krienitz (eds.). Süßwasserflora von Mitteleuropa. 19/2. London, Elsevier.

Lopes, M.R.M.; Bicudo, C.E.M. \& Ferragut, C. 2005. Short term spatial and temporal variation of phytoplankton in a shallow tropical oligotrophic reservoir, southeast Brazil. Hydrobiologia 542: 235-247.

Mackereth, J.J.H.; Heron, J. \& Talling, J.F. 1978. Water analysis: some revised methods for limnologists. Scientific Publication $\mathrm{n}^{\circ} 36$, London, Freshwater Biological Association.

Margalef, R. 1983. Limnologia. Barcelona, Omega, S.A.

Marinho, M.M. \& Huszar, V.L.M. 2002. Nutrient availability and physical conditions as controlling factors of phytoplankton composition and biomass in a tropical reservoir (Southeastern Brazil). Archiv für Hydrobiologie 153: 443-468.

McCune, B. \& Mefford, M.J. 1999. PC-ORD for Windows: multivariate analysis of ecological data. $\mathrm{MjM}$ Software, Oregon, Gleneden Beach.

Melo, S. \& Huszar, V.L.M. 2000. Phytoplankton in an Amazonian flood-plain lake (Lago Batata, Brazil): diel variation and species strategies. Journal of Plankton Research 22: 63-76.

Molica, R.J.R.; Oliveira, E.J.A.; Carvalho, P.V.V.C.; Costa, A.N.S.F.; Cunha, M.C.C.; Melo, G.L. \& Azevedo, S.M.F.O. 2005. Occurrence of saxitoxins and an anatoxin-a(s)-like anticholinesterase in a Brazilian drinking water supply. Harmful Algae 4: 743-753.

Moura, A.N.; Bittencourt-Oliveira, M.C.; Dantas, E.W. \& Arruda Neto, J.D.T. 2007. Phytoplanktonic Associations: A Tool to Understand Dominance Events in a Tropical Brazilian Reservoir. Acta Botanica Brasilica 21: 641-648.

Negro, A.I.; De Hoyos, C. \& Veja, J. C. 2000. Phytoplankton structure and dynamics in Lake Sanabria and Valparaíso reservoir (NW Spain). Hydrobiologia 424: 25-37.

Padisák, J. 1997. Cylindrospermopsis raciborskii (Woloszynska) Seenayya et Subba Raju, an expanding, highly adaptative cyanobacterium: worldwide distribution and review of its ecology. Algological Studies 107: 563-593.

Padisák, J. \& Reynolds, C.S. 1998. Selection of phytoplankton assemblages in lake Balaton, Hungary, in response to eutrophication and restoration measures, with special reference to the cyanoprokariotes. Hydrobiologia 384: 41-53.

Ramirez, J.J. \& Bicudo, C.E.M. 2002. Variation of climatic and physical co-determinants of phytoplankton community in four nictemeral sampling days in a shallow tropical reservoir, southeastern Brazil. Brazilian Journal of Biology 62: 1-14.

Ramirez, J.J. \& Bicudo, C.E.M. 2005. Diurnal and spatial (vertical) dynamics of nutrient $(\mathrm{N}, \mathrm{P}, \mathrm{Si}$ ) in four sampling days (summer, fall, winter and spring) in a tropical shallow reservoir and their relationships with the phytoplankton community. Brazilian Journal of Biology 65: 141-157.

Reynolds, C.S. 1984. Phytoplankton periodicity; the interactions of form, function and environmental variability. Freshwater Biology 14: 111-142.
Reynolds, C.S. 1997. Vegetation processes in the pelagic: a model for ecosystem theory. Oldendorf, Ecology Institute.

Reynolds, C.S. \& Walsby, A.E. 1975. Water blooms. Biological Reviews 50: 437-81.

Reynolds, C.S.; Olivier, R.L. \& Walsby, A.E. 1987. Cyanobacterial dominance: the role of buoyancy regulation in dynamic lake environments. New Zeland Journal of Marine and Freshwater Research 21: 379-390.

Romo, S. \& Miracle, M.R. 1995. Diversity of the phytoplankton assemblages of a polimitic hypertrophic lake. Archiv für Hydrobiologie 132: 363-384.

Round, F.E.; Crawford, R.M. \& Mann, D.G 1990. The Diatoms. Biological \& morphology of the genera. Cambridge, Cambridge University Press.

Saker, M.L.; Neilan, B.A. \& Griffiths, D.J. 1999. Two morphological forms of Cylindrospermopsis raciborskii (Cyanobacteria) isolated from Solomon Dam, Palm Island, Queensland. Journal of Phycology 35: 599-606.

Sant'Anna, C.L. 1984. Chlorococcales (Chlorophyceae) do Estado de São Paulo, Brasil. Bibliotheca Phycologica 67: 1-348.

Shapiro, J. 1990. Current beliefs regarding dominance of blue-greens: the case for importance of $\mathrm{CO}_{2}$ and $\mathrm{pH}$. Verhandlungen der Internationalen Vereinigung für Theoretische und Angewandte Limnologie 24: 38-54.

Smith, V.H. 1983. Low nitrogen to phosphorus ratios favor dominance by blue-green algae in lake phytoplankton. Science 221: 669-671.

SRH (Secretaria de Recursos Hídricos de Pernambuco) 2000. Plano Estadual de Recursos Hídricos do Estado de Pernambuco - Documento Síntese, Recife.

Strahler, A. 1986. Geografia Física. Barcelona, Omega, S.A.

Straskraba, M. \& Tundisi, J.G. 2000. Diretrizes para o gerenciamento de lagos: gerenciamento da qualidade dos lagos. São Carlos, ILEC/IIE.

Strickland, J.D. \& Parsons, T.R. 1965. A manual of sea water analysis. Bulletin of the Fisheries Research Board of Canada 125: $1-185$.

Sommer, U. 1988. Growth and survival strategies of plankton succession. Pp 57-106. In: U. Sommer (ed.). Plankton Ecology - Succession in Plankton Communities. Springer Series of Contemporary Bioscience.

Tilman, D. \& Kilham, S.S. 1976. Phosphate and silicate uptake and growth kinetics of the diatoms Asterionella formosa and Cyclotella meneghiniana in batch and semi-continuous culture. Journal of Phycology 12: 375-383.

Tucci, A. \& Sant'Anna, C.L. 2003. Cylindrospermopsis raciborskii (Woloszynska) Seenayya \& Subba Raju (Cyanobacteria): variação semanal e relações com fatores ambientais em um reservatório eutrófico, São Paulo, SP, Brasil. Revista Brasileira de Botânica 26: 97-112.

Valderrama, G.C. 1981. The simultaneous analysis of total nitrogen and total phosphorus in natural waters. Marine Chemistry 10: $109-122$.

Watson, S.B.; McCauley, E. \& Downing, J.A. 1997. Patterns in phytoplankton taxonomic composition across temperate lakes of differing nutrient status. Limnology and Oceanography 42: 487-495. 